\title{
Determinación de la Dominancia Coronaria en Población Mestiza Colombiana. Un Estudio Anatómico Directo
}

\author{
Coronary's Dominance Determination in Racially Mixed Colombian Population. A Direct Anatomical Study
}

"Luis Ernesto Ballesteros Acuña; **Edgar Giovanni Corzo Gómez \& *Bladimir Saldarriaga Tellez

BALleSTEROS, L. E.; CORZO, E. G. \& SALDARRIAGA, B. Determinación de la dominancia coronaria en población mestiza colombiana. Un estudio anatómico directo. Int. J. Morphol., 25(3):483-491, 2007.

RESUMEN: Los estudios de dominancia coronaria realizados en diversos grupos poblacionales han reportado gran variabilidad. El propósito de este trabajo fue determinar la dominancia coronaria en un grupo de mestizos colombianos. Se evaluaron los corazones obtenidos de 154 cadáveres frescos obtenidos como material de autopsia. Las arterias coronarias fueron sometidas a proceso de inyección con resinas sintéticas e impregnadas en solución de $\mathrm{KOH}$ al 15\% para liberar la grasa subepicárdica. Se observó dominancia coronaria derecha en el 83,7\% de los especímenes cardiacos, correspondiendo la expresión mayoritaria de éstos, a la dominancia moderada con el 42,8\%. La circulación balanceada se observó en el 9,2\% y la dominancia izquierda en el 7,2\%, con una mayor frecuencia del subgrupo II en un 3,3\% de los casos. La dominancia derecha se presentó con mayor frecuencia en mujeres pero no fue estadísticamente significativa $(p=0,26)$. La rama circunfleja fue de expresión corta en 143 corazones $(92,8 \%)$ y de expresión larga en el 7,2\%. La arteria coronaria derecha finalizó entre la cruz cardiaca y el margen izquierdo en el 81,8\% y con menor frecuencia en el margen izquierdo en un $1,9 \%$. El calibre de la arteria coronaria derecha en los corazones con dominancia derecha fue de 3,21 mm (SD 0,53) y en los corazones con dominancia izquierda fue de $2,67 \mathrm{~mm}$ (SD 0,30), existiendo una diferencia estadísticamente significativa $(\mathrm{p}=0,0066)$. Hubo un predominio de la dominancia coronaria derecha de expresión moderada, arterias circunflejas cortas y arterias coronarias derechas con mayor calibre en los especímenes con dominancia derecha.

PALABRAS CLAVE: Corazón; Circulación coronaria; Dominancia; Circulación balanceada; Calibre arterial.

\section{INTRODUCCIÓN}

La irrigación del corazón proviene de las arterias coronarias derecha (ACD) e izquierda (ACI), las cuales se originan de la parte ascendente de la aorta a la altura de los senos aórticos derecho e izquierdo. Existe gran variabilidad en la expresión morfológica de estos vasos en relación al calibre, trayectoria, presencia de colaterales, como la rama lateral o posterolateral derecha, y a los territorios ventriculares irrigados, de los cuales se ha fundamentado el concepto de dominancia coronaria derecha o izquierda, dependiendo de cual arteria coronaria da origen a la rama interventricular posterior o de aquella que irrigue la mayor parte de la pared ventricular posterior izquierda. (Penthe et al., 1976; Cavalcanti et al., 1995; Falci et al., 1996 ; Ilia et al., 2001).

La concepción de dominancia coronaria fue propuesta por Schlesinger (1940). Dominancia derecha cuando la arteria coronaria derecha irriga la superficie posterior del

\footnotetext{
* Profesor Asociado Universidad Industrial de Santander, Colombia.

***Profesor Asistente, Universidad Autónoma de Bucaramanga, Colombia.

* Profesor Asociado, Universidad Autónoma de Bucaramanga, Colombia.
}

ventrículo derecho, da origen a la rama interventricular posterior y continúa más allá de la cruz cardiaca para irrigar una porción del ventrículo izquierdo. Balanceada cuando la arteria coronaria derecha irriga el ventrículo derecho y la porción posterior del septum interventricular a través de la rama interventricular posterior (RIP), mientras la arteria coronaria izquierda irriga el ventrículo izquierdo finalizando en la cruz cardiaca. Dominancia izquierda, si la arteria coronaria izquierda irriga la cara posterior del ventrículo izquierdo, el segmento posterior del septum interventricular y/o la pared posterior del ventrículo derecho.

En razón a que el concepto de dominancia coronaria izquierda era muy amplio y teniendo en cuenta la variabilidad de este tipo de irrigación, DiDio \& Wakefield (1975) propusieron un subgrupo I, cuando ambas arterias coronarias derecha e izquierda alcanzan la cruz cardiaca y terminan 
como ramas interventriculares posteriores paralelas; subgrupo II, cuando el ventrículo izquierdo y la totalidad del septum interventricular es irrigado por la arteria coronaria izquierda, sólo hay una rama interventricular posterior que se origina de la rama circunfleja, rama de la arteria coronaria izquierda; y un subgrupo III, si la RIP y la(s) rama(s) ventricular(es) posterior(es) derecha(s) se originan de la arteria coronaria izquierda dando así la irrigación para el ventrículo izquierdo, para la totalidad del septum interventricular y para una parte de la pared posterior del ventrículo derecho.

La importancia del conocimiento de las variaciones morfológicas de las arterias coronarias, de manera particular la dominancia coronaria, radica, además del concepto académico, en su impacto sobre la cirugía cardiaca, los procedimientos hemodinámicos, en la etiología de las arritmias derivadas de la enfermedad coronaria oclusiva y en el manejo del trauma cardiaco (Baptista et al., 1989; Reig et al., 1993; Kalpana, 2003).

La determinación de la dominancia coronaria, ha sido descrita mediante técnicas de disección clásica, inyeccióncorrosión o estudios radiográficos, en otros grupos poblacionales, algunos con una clara orientación básico-clínica (Ahmed et al., 1972; Llerena et al., 1996; Loukas et al., 2006). La ausencia de este tipo de información en población mestiza colombiana le da pertinencia a la realización del presente estudio, desarrollado en material cadavérico fresco, con el propósito de obtener información propia de referencia.

\section{MATERIAL Y MÉTODO}

En este estudio no probabilístico y descriptivo transversal se evaluaron los corazones obtenidos de 154 cadáveres frescos (125 hombres; 29 mujeres ) de individuos fallecidos violentamente, a quienes se les practicó necropsia en el Instituto de Medicina Legal y de Ciencias Forenses de Bucaramanga, Colombia.

Se obtuvo una muestra por conveniencia, que cumpliera con los siguientes criterios de inclusión: Corazones de sujetos masculinos y femeninos de raza mestiza (se consideraron mestizos a los individuos que presentaron mezcla fenotípica de los grupos raciales caucasoide, negroide y mongoloide), sin signos de patología o trauma cardiaco. Las arterias coronarias fueron sometidas a proceso de inyección con resina sintética (palatal GP41L 80\% y estireno 20\%) a una presión de $120 \mathrm{~mm}$ de mercurio, luego los corazones se colocaron en solución de $\mathrm{KOH}$ al $15 \%$ por cinco minutos para liberar la grasa subepicárdica. (Nerantzis et al., 1978)
Se realizaron mediciones del calibre de las arterias coronarias y sus ramas a 5 milímetros de sus orígenes, con calibrador electrónico (Mitutoyo); se registraron trayectorias, frecuencias de las coronarias y sus colaterales. De cada una de las piezas evaluadas se obtuvieron registros fotográficos.

Se determinó el nivel de finalización de las arterias coronarias izquierda y derecha y complementando los patrones establecidos por Schlesinger y DiDio \& Wakefield, se establecieron los criterios para la dominancia coronaria derecha así: leve si alcanza el tercio derecho del ventrículo izquierdo; moderada cuando llega hasta el tercio medio; y prominente, cuando alcanza el tercio lateral o el margen obtuso del corazón.

Se determinó el sitio de finalización de la RIP y se clasificó como larga cuando alcanzó el tercio inferior del surco homónimo, el ápex e incluso el tercio inferior del surco interventricular anterior; y corta, si finalizó en los segmentos superior o medio del surco interventricular posterior (SIVP).

Las variables continuas se describieron con sus promedios y desviación estándar, las variables nominales con sus proporciones. Se realizaron las pruebas estadísticas de chi $\left(\mathrm{X}^{2}\right)$ cuadrado y test de student aceptando un error alfa hasta de un 5\%. La base de datos se digitó en Excel y los análisis estadísticos se realizaron en STATA 8.0.

\section{RESULTADOS}

La edad promedio de los individuos en que se obtuvieron los especimenes cardíacos, fue de 31,4 años (16-75 años), y el peso promedio fue de 291,2 g DS 45,5 (hombres 300,7 DS 58; mujeres 248,4 DS 61,1).

La rama circunfleja ( $\mathrm{RCX})$ fue corta (terminando antes o en la cruz cardiaca) en 143 corazones $(92,8 \%)$ y fue larga, alcanzando el surco interventricular posterior o dando ramas ventriculares posteriores derechas en 11 corazones $(7,2 \%)$, (Tabla I).

En la mayoría de las piezas anatómicas, la ACD finalizó entre la cruz y el margen izquierdo, irrigando algunos de los segmentos de la superficie diafragmática del ventrículo izquierdo $(81,8 \%)$ y con menor frecuencia, $(1,9 \%)$ finalizó en el margen izquierdo. (Tabla I).

En el 83,7\% de los especimenes cardiacos se observó dominancia coronaria derecha (Fig.1), correspondiendo la expresión mayoritaria a la dominancia moderada con el $42,8 \%$ ( Fig. 2). La circulación balanceada se observó en el 

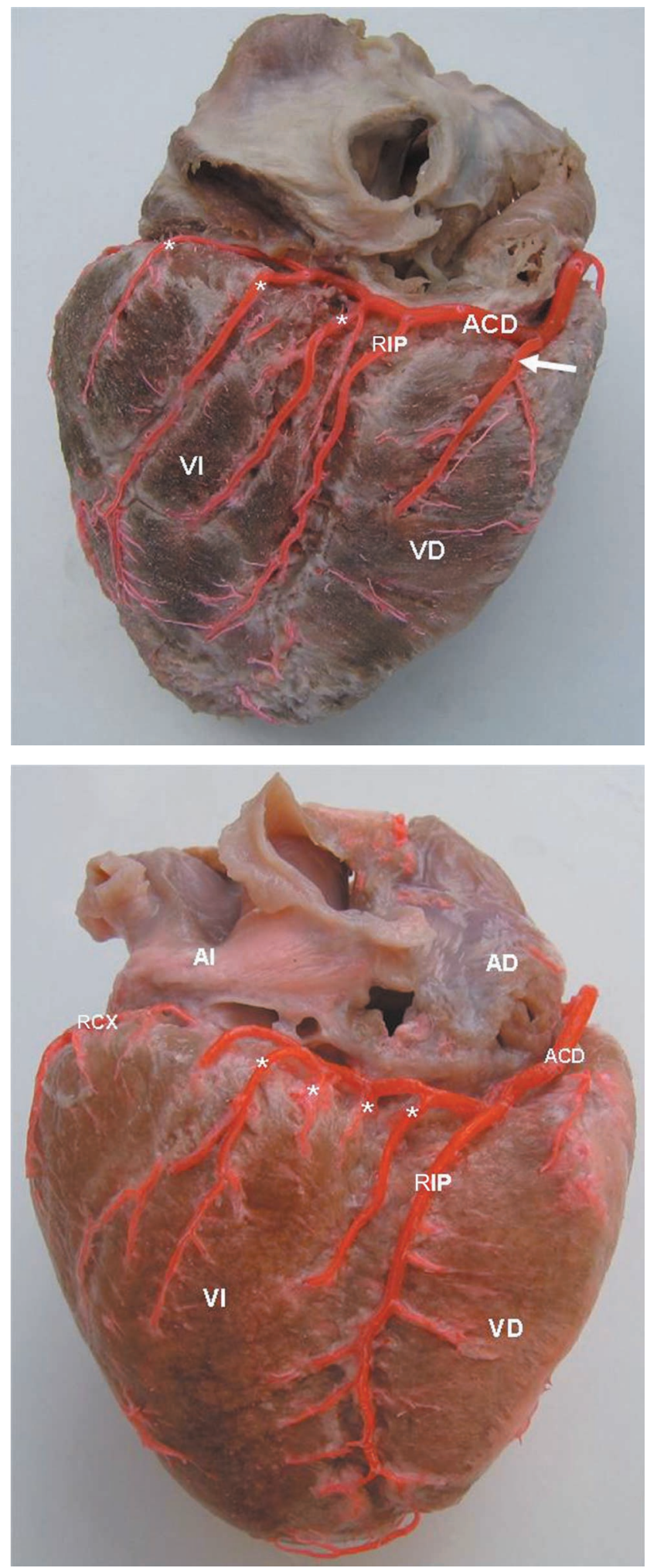

Fig. 2. Dominancia derecha moderada. Cara diafragmática del corazón. VI. Ventrículo izquierdo; VD. Ventrículo derecho. RIP. Rama interventricular posterior; ACD. Arteria coronaria derecha. *Ramas ventriculares posteriores izquierdas; AD. Atrio derecho; AI. Atrio izquierdo; RCX. Rama circunfleja.

Fig.1. Dominancia derecha prominente. Cara diafragmática del corazón. VI. Ventrículo izquierdo; VD. ventrículo derecho; ACD. Arteria coronaria derecha; RIP. Rama interventricular posterior. * Ramas ventriculares posteriores izquierdas; Flecha. Rama posterolateral derecha.

9,2\% (Fig. 3); y la dominancia izquierda en el 7,2\% (Fig. 4), con una mayor frecuencia del subgrupo II con el 3,3\% de los casos. (Tabla II) (Fig. 5). La dominancia derecha se presentó con mayor frecuencia en mujeres (Tabla III), pero no fue estadísticamente significativa $(\mathrm{p}=0,26)$.

La RIP fue larga en el 70,6\%; y corta en el 29,4\% de los casos (Tabla IV). La RIP larga se observó con mayor frecuencia en mujeres $(72,5 \%)$ que en hombres $(70,6 \%)$ sin que fuera esta diferencia estadísticamente significativa $(\mathrm{p}=0,98)$.

El segmento más frecuente de finalización de la rama interventricular anterior (RIA) fue el tercio inferior del surco interventricular posterior, en el $63,6 \%$ de los casos, correspondiendo a los hombres el $61,7 \%$ y a las mujeres el 71,4\% (Tabla V); esta diferencia fue estadísticamente significativa $(\mathrm{p}=0,00)$ con relación a la finalización en el ápex cardiaco $(27,6 \%$ de las muestras).

Tabla I. Tipo y terminación de la ramas circunfleja y arteria coronaria derecha en 154 corazones de individuos mestizos colombianos, de ambos sexos.

\begin{tabular}{lrr}
\hline Tipo y terminación de la rama circunfleja & $\mathrm{n}$ & $\%$ \\
& 154 & 100,0 \\
\hline Rama circunfleja larga & 11 & 7,2 \\
En el surco interventricular posterior & 8 & 5,2 \\
Dando ramas interventriculares posteriores derechas & 3 & 2,0 \\
Rama circunfleja corta & 143 & 92,8 \\
Como marginal izquierda & 39 & 25,3 \\
Ent re el margen izquierdo y la cruz cardiaca & 90 & 58,4 \\
Enla cruz cardiaca & 14 & 9,1 \\
& & \\
Terminación de la arteria coronaria derecha & 154 & 100,0 \\
Entre el marg en derecho y la cruz cardiaca & 11 & 7,2 \\
Enla cruz cardiaca y da la r. interventricular posterior & 14 & 9,1 \\
Ent re la cruz cardiaca y el margen izquierdo & 126 & 81,8 \\
En el margen izquierdo & 3 & 1,9 \\
\hline
\end{tabular}

Tabla II. Cuantificación de la dominancia coronaria en 154 corazones de individuos mestizos colombianos, de ambos sexos.

\begin{tabular}{lrr}
\hline Distribución de la dominancia coronaria & $\mathrm{n}$ & $\%$ \\
\hline Derecha & 154 & 100,0 \\
Leve & 129 & 83,7 \\
Moderada & 52 & 33,7 \\
Prominente & 66 & 42,8 \\
Balanceada & 11 & 7,2 \\
Izquierda & 14 & 9,2 \\
Subgrupo I & 11 & 7,1 \\
Subgrupo II & 3 & 1.9 \\
Subgrupo III & 5 & 3,3 \\
\hline
\end{tabular}




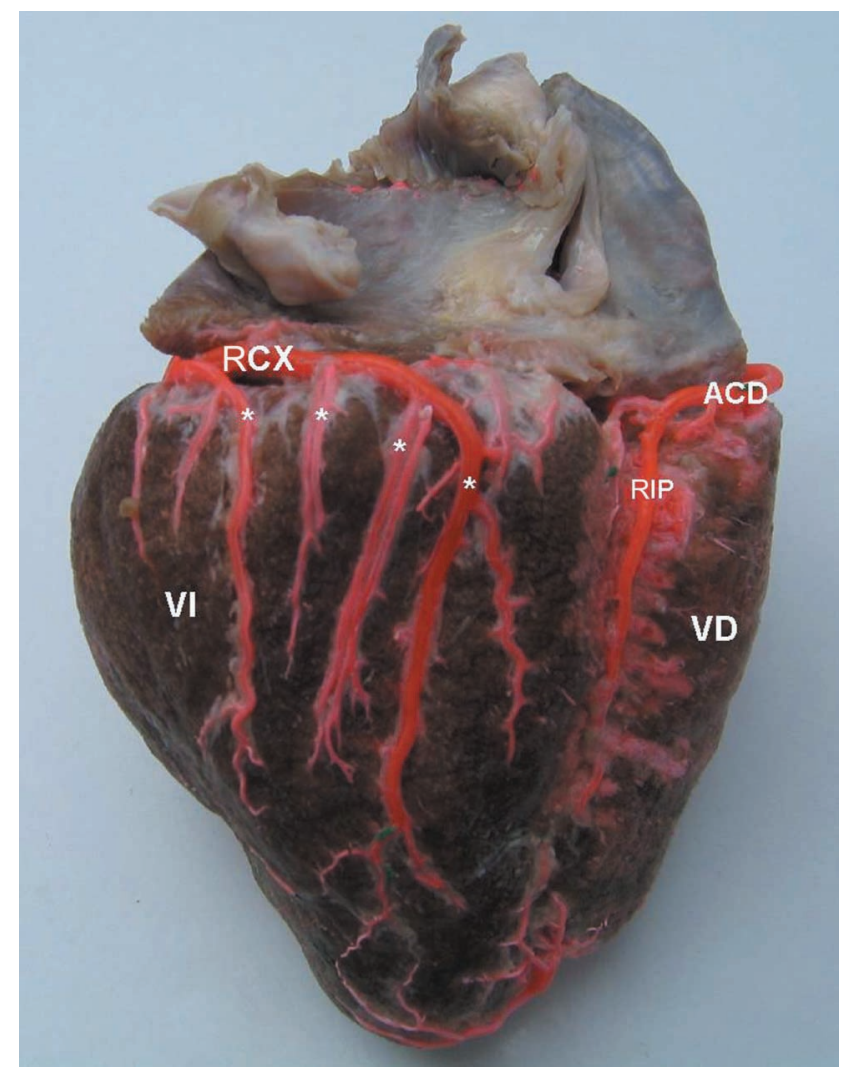

Fig. 3. Circulación balanceada. Cara diafragmática del corazón. VI. Ventrículo izquierdo. VD. Ventrículo derecho. RIP. Rama interventricular posterior. ACD. Arteria coronaria derecha. *Ramas ventriculares posteriores izquierdas; RCX. Rama circunfleja.

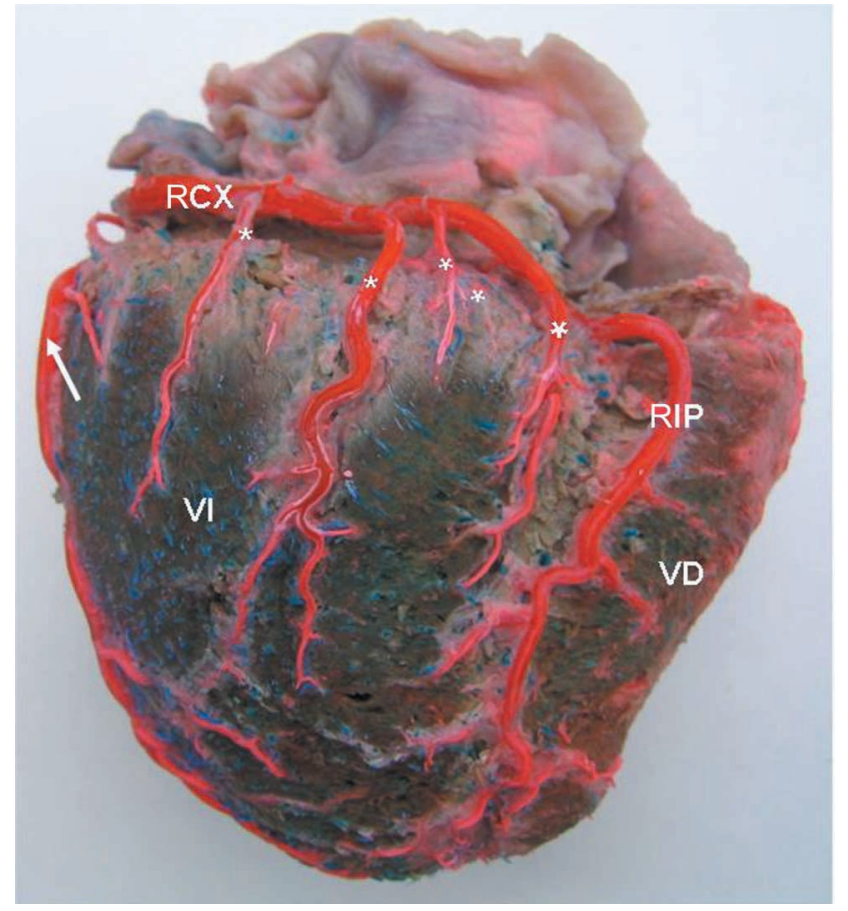

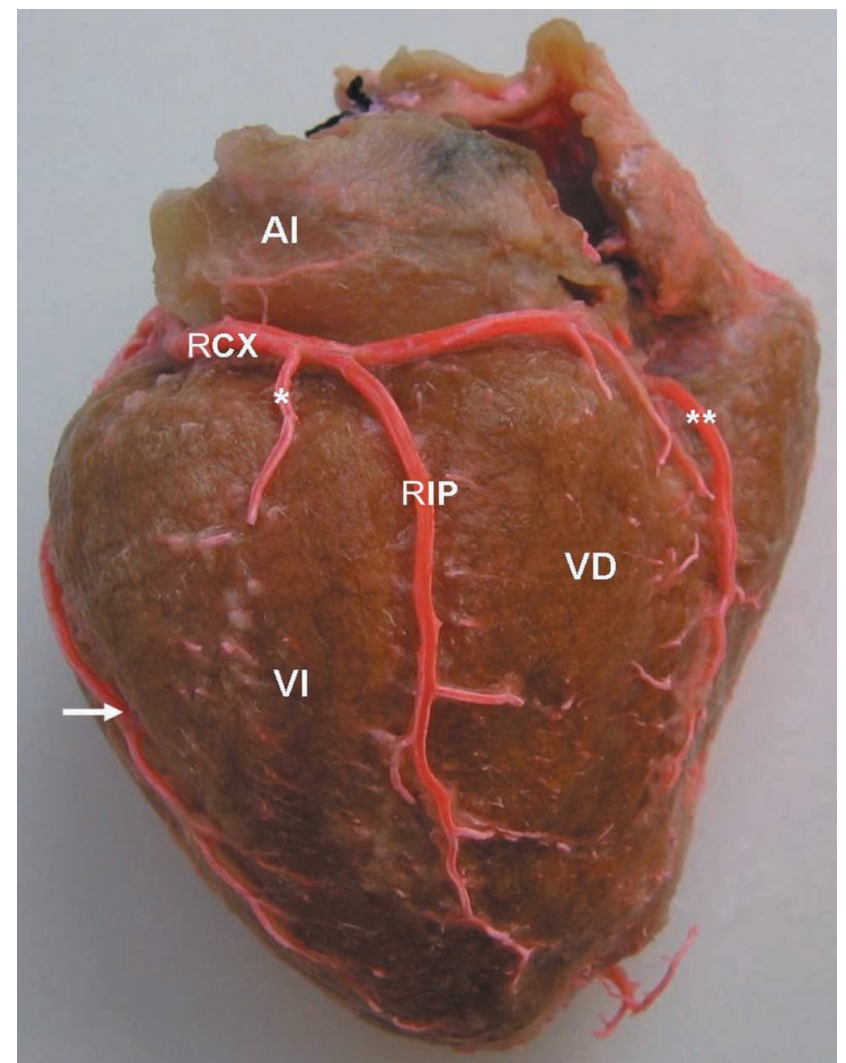

Fig. 4. Dominancia izquierda subgrupo III. Cara diafragmática del corazón. VI. Ventrículo izquierdo; VD. Ventrículo derecho; RIP. Rama interventricular posterior; RCX. Rama circunfleja; *Rama ventricular posterior izquierda. **Rama ventricular posterior derecha; AI. Atrio izquierdo; Flecha. Rama marginal izquierda.

El calibre de la ACI fue de 3,58 mm y el de la ACD fue de 3,18 mm. Igualmente, dentro de las ramas de la ACI, la RIA fue de mayor diámetro que la $\operatorname{RCX}(2,94$ y 2,71 mm respectivamente). El calibre de la ACD en los corazones con dominancia derecha fue de 3,21 mm SD 0,53 y en los corazones con dominancia izquierda fue de 2,67 mm SD 0,30 existiendo una diferencia estadísticamente significativa. $(\mathrm{p}=$ 0,0066).

La ACI presentó un calibre de 3,40 mm SD 0,86 en los casos con dominancia coronaria izquierda y de $3,54 \mathrm{~mm}$ SD 0,55 en la dominancia derecha $(p=0,1849)$. Las RIAs presentaron un calibre de 2,92 mm SD 0,47 en los casos de dominancia derecha y de 2,97 mm SD 0,63 en los de dominancia izquierda $(p=0,28)$. El calibre de las RCX fue de 2,66 mm SD 0,48 y de 2,83 mm SD 0,87 para las dominancias derecha e izquierda, respectivamente. $(\mathrm{p}=0,0141)$.

Fig. 5. Dominancia izquierda subgrupo II. Cara diafragmática del corazón. VI. Ventrículo izquierdo; VD. Ventrículo derecho; RIP. Rama interventricular posterior. RCX. Rama circunfleja. *Ramas ventriculares posteriores izquierdas; Flecha. Rama marginal izquierda. 
Tabla III. Frecuencia del tipo de dominancia coronaria según el sexo en 154 corazones de individuos mestizos colombianos.

\begin{tabular}{lrrrrrrr}
\hline Tipo de dominancia & \multicolumn{2}{c}{ Masculino } & \multicolumn{2}{c}{ Femenino } & & Total & Test p \\
\hline & $\mathrm{n}$ & $\%$ & $\mathrm{n}$ & $\%$ & $\mathrm{n}$ & \multicolumn{1}{c}{$\%$} \\
\hline Derecha & 102 & 81,6 & 27 & 93,1 & 129 & 83,7 & 0.26 \\
Circulación balanceada & 14 & 11,2 & 0 & 0,0 & 14 & 9,1 & 0.20 \\
Izquierda & 9 & 7,2 & 2 & 6,9 & 11 & 7,2 & 0.85 \\
\hline
\end{tabular}

Tabla IV. Finalización de la rama interventricular posterior según el sexo en 154 corazones de individuos mestizos colombianos.

\begin{tabular}{|c|c|c|c|c|c|c|c|}
\hline \multirow{2}{*}{\multicolumn{2}{|c|}{$\begin{array}{c}\text { Lugar de } \\
\text { terminación de la } \\
\text { RIP }\end{array}$}} & \multicolumn{2}{|c|}{ Corta $45(29,4 \%)$} & \multicolumn{3}{|c|}{ Larga $108(70,6 \%)$} & \multirow[t]{2}{*}{ TOTAL } \\
\hline & & $\begin{array}{l}\text { Segmento } \\
\text { superior del } \\
\text { SIVP } *\end{array}$ & $\begin{array}{l}\text { Segmento medio } \\
\text { del SIVP } *\end{array}$ & $\begin{array}{c}\text { Segmento } \\
\text { inferior del SIVP } \\
*\end{array}$ & \multirow{2}{*}{$\begin{array}{c}\text { En el ápex } \\
\text { cardíaco } \\
31\end{array}$} & En el SI & \\
\hline Hombres & $\mathrm{n}$ & 12 & 25 & 55 & & 1 & 124 \\
\hline & $\%$ & 9,7 & 20,2 & 44,4 & 25 & 0,7 & 100 \\
\hline Mujeres & $\mathrm{n}$ & 3 & 5 & 19 & 1 & 1 & 29 \\
\hline & $\%$ & 10,3 & 17,2 & 65,5 & 3,5 & 3,5 & 100 \\
\hline Total & $\mathrm{n}$ & 15 & 30 & 74 & 32 & 2 & 153 \\
\hline & $\%$ & 9,8 & 19,6 & 48,4 & 20,9 & 1,3 & 100 \\
\hline
\end{tabular}

* SIVP: surco interventricular posterior. ** SIVA: surco interventricular anterior.

Tabla V. Terminación de la rama interventricular anterior de acuerdo al sexo en 154 corazones de individuos mestizos colombianos.

\begin{tabular}{lccccccccc}
\hline & $\begin{array}{c}\text { Antes del ápex } \\
\text { cardiaco }\end{array}$ & \multicolumn{2}{c}{ En el ápex cardiaco } & \multicolumn{2}{c}{$\begin{array}{c}\text { En el tercio inferior } \\
\text { del SIVP }\end{array}$} & \multicolumn{2}{c}{$\begin{array}{c}\text { En el tercio } \\
\text { intermedio del SIVP }\end{array}$} & \multicolumn{2}{c}{$\begin{array}{c}\text { Entre el tercio medio } \\
\text { e inferior del SIVP }\end{array}$} \\
\hline & $\mathrm{n}$ & $\%$ & $\mathrm{n}$ & $\%$ & $\mathrm{n}$ & $\%$ & $\mathrm{n}$ & $\%$ & $\mathrm{n}$ \\
Hombre & 1 & 0,8 & 36 & 29,3 & 76 & 61,7 & 6 & 4,9 & 4 \\
Mujer & 1 & 3,6 & 6 & 21,4 & 20 & 71,4 & 0 & 0 & 1 \\
Total & 2 & 1,3 & 42 & 27,8 & 96 & 63,6 & 6 & 4,0 & 5 \\
\hline
\end{tabular}

\section{DISCUSIÓN}

Para determinar el tipo dominante de la circulación del corazón, se requiere evaluar previamente las dimensiones de la RCX y de la ACD, comprometidas en la irrigación de la cara diafragmática. La frecuencia de la RCX corta hallada en este trabajo $(92,85 \%)$, es similar al reporte de Mouchet (1933), pero más alto al descrito por otros autores: Banchi (1904) (70\%), James (1961) (81\%), Baptista et al. (1990) $(86,4 \%)$ y Kalpana (83\%). Una incidencia baja fue señalada por Reig et al. (1987) (53\%). Concomi-tantemente, la frecuencia del tipo de RCX larga en nuestro trabajo es más bajo que el reportado en estudios previos: Baroldi \& Scomazzoni (1967) (21\%), Taylor (1977) (23\%), Blunk \& DiDio (1971) (20\%), Baptista et al. (1990) (13,6\%), Kalpana (17\%). La alta frecuencia de la presentación de una RCX corta se correlaciona con la baja incidencia de dominancia coronaria izquierda observada en este trabajo.
Las RCX más cortas terminan como ramas marginales, situación descrita por Baroldi \& Scomazzoni en 16\%, De Paula (1972) 21\%, Baptista et al. (1990) 9.1\%, Kalpana en $13 \%$ y en nuestro trabajo en $25,3 \%$. En este número elevado de casos se observó que las ramas marginales, además de irrigar los segmentos de la cara obtusa del corazón, a través de ramas colaterales dispuestas en trayectorias horizontales u oblicuas, participaban con las ramas posteriores del ventrículo izquierdo de la ACD en la irrigación de los segmentos laterales y medios de la cara posterior del ventrículo izquierdo.

La más frecuente finalización de la ACD en nuestra serie $(81,8 \%)$ fue a través de ramas posteriores del ventrículo izquierdo. Este porcentaje es similar al reportado por Baroldi \& Scomazzoni y más alto que los descritos por Blunk \& 
DiDio (78\%), Taylor (76\%), Baptista et al. (71,6\%) y Kalpana (76\%). James (1961) reportó 88\%. En este trabajo se observó la ACD corta, finalizando como rama posterior del ventrículo derecho en el 7.2\% de los casos, cifra que es concordante con los reportes previos (James (1961), Taylor, Baptista et al.; Kalpana). Al finalizar la ACD en la cara diafragmática del ventrículo derecho a través de pequeñas ramas, la adecuada irrigación de este territorio requiere de la suplencia de ramas de la RCX y de la RIA, que traspasando el ápex cardiaco debe distribuirse en el segmento postero-inferior o incluso en el medio.

El espectro de presentación de dominancia de la circulación cardiaca, señalada por múltiples autores, de acuerdo a los criterios señalados por Schlesinger para determinar la dominancia coronaria, es muy amplia: Frecuencias de dominancia derecha alta con rango de 85 a 90\% (James, 1965, Penthe et al. y Nerantzis et al., 1996); Intermedia con rango de 70 al 84\% (DiDio \& Wakefield; Blunk \& DiDio; Taylor; Hadziselimovic, 1981; Sahni \&
Jit, 1990; Llerena et al.; Kalpana y Pessa et al., 2004), en la cual se halla este estudio, y dominancia baja con rango del 48 al 70\%( Schlesinger; Ortale et al., 2004; Kaimkhani et al., 2005; Cavalcanti et al. y Loukas et al).

Corresponde de igual manera tres rangos de expresión para la dominancia coronaria izquierda; una baja, en la cual se incluyó nuestro trabajo con el 5 al 12\% de los casos (Banchi; Ortale et al.; Zbigniew \& Mikusek, 2000 y Kalpana; una intermedia con rango del 13 el 20\% (Schlesinger; Blunk \& Didio; Sahni \& Jit y Kaimkhani et al. ), y una alta con una frecuencia de expresión mayor al 20\% (Baroldi \& Scomazzoni; May, 1960. Para la circulación balanceada, caracterizada porque la ACD termina como rama interventricular posterior, sin llegar a irrigar la pared posterior del ventrículo izquierdo, los reportes de la literatura presentan frecuencias con rango del 7 al 26\%. (Polacek \& Zechmeister, 1968 ; Llerena et al.; Ortale et al.; Zbigniew, \& Mikusek y Loukas et al. ). La Tabla VI muestra la distribución de los diversos tipos de circulación coronaria de acuerdo a varios autores.

Tabla VI. Distribución de los diversos tipos de dominancia coronaria de acuerdo a varios autores.

\begin{tabular}{lccc}
\hline Autor año & $\begin{array}{c}\text { Dominancia } \\
\text { derecha }\end{array}$ & $\begin{array}{c}\text { Circulación } \\
\text { Balanceada }\end{array}$ & $\begin{array}{c}\text { Dominancia } \\
\text { izquierda }\end{array}$ \\
\hline Banchi, 1904 & 87 & - & 13 \\
Schlesinger, 1940 & 48 & 34 & 18 \\
May, 1960 & 55 & 9 & 36 \\
James, 1965 & 90 & - & 10 \\
Polacek \& Zechmeister, 1968 & 68 & 21 & 11 \\
Blunk \& DiDio, 1971 & 78 & 2 & 20 \\
DiDio \& Wakefield, 1975 & 73,5 & 7,1 & 19,4 \\
Taylor, 1977 & 76,4 & 11,3 & 12,2 \\
Hadziselimovic, 1981 & 70 & 20 & 10 \\
Baptista \& DiDio, 1989 & 71,6 & 17,3 & 11,1 \\
Sahni \& Jit, 1990 & 70,4 & 11,5 & 18,1 \\
Cavalcanti et al., 1995 & 69,1 & 19,1 & 11,8 \\
Llerena et al., 1996 & 84,6 & 6,4 & 9 \\
Zbigniew \& Mikusek, 2000 & 67,5 & 23,9 & 8,6 \\
Kalpana, 2003 & 84 & 6 & 10 \\
Ortale et al., 2004 & 62,5 & 25 & 12,5 \\
Pessa et al., 2004 & 81,2 & 16,5 & 2,4 \\
Kaimkhani et al., 2005 & 64,5 & 24,5 & 15 \\
Loukas et al., 2006 & 55 & 12 & 33 \\
\hline
\end{tabular}


En relación al sexo, existe controversia sobre el tipo de circulación coronaria que es más frecuente. DiDio \& Wakefield, Baptista et al., 1989, y Sahni \& Jit reportan la dominancia derecha más frecuente en hombres, mientras que en las mujeres es frecuente la dominancia izquierda. En nuestro trabajo, en concordancia con Schlesinger, James (1961), Ahmed et al. y Kaimkhani et al. se observó que la dominancia izquierda presenta mayor incidencia en hombres, sin que sea esta diferencia estadísticamente significativa.

Las diferencias amplias en los porcentajes, de los diversos tipos de dominancia coronaria y las relaciones con respecto al género, presentados en los trabajos, se debe a varios factores tales como el tamaño de las muestras, valoración imagenológica o de disección directa y los criterios de definición de dominancia coronaria. Los estudios anatómicos directos presentan (por las dificultad de obtener un número adecuado de piezas procedentes de sujetos femeninos), evidentes limitaciones de correlacionar los resultados con relación al género.

DiDio \& Wakefield y Ortale et al., han modificado los criterios de Schlesinger para determinar la dominancia coronaria, al señalar con mayor especificidad la manera como la RCX y la ACD irrigan la cara diafragmática de los ventrículos izquierdo y derecho. DiDio \& Wakefield reportaron dominancia izquierda en $19.4 \%$ de los casos, correspondiendo al subgrupo I $6,1 \%$, al subgrupo II $10,2 \%$ y al subgrupo III 3,1\%. En nuestra serie se encontró el subgrupo II $(3,3 \%)$ como el más frecuente, con cerca del $50 \%$ de los casos de dominancia izquierda; estos resultados son similares a los descritos por DiDio \& Wakefield y revelan que en la dominancia izquierda, la RCX termina con gran frecuencia como RIVP y en un número escaso como rama ventricular posterior derecha.

Ortale et al. dividieron la cara posterior de los ventrículos en ocho cuadrantes para obtener mejor precisión sobre los territorios irrigados por la RCX y ACD. Cuando la RIP era corta y los cuadrantes mediales eran suplidos por la RIA, consideraban la muestra como de irrigación balanceada. Este criterio elevó en su estudio el porcentaje de este tipo de irrigación al $25 \%$. Este autor reportó mayor incidencia $(47,5 \%$ del total de $62,5 \%)$ al tipo de dominancia derecha moderada, resultado similar al obtenido en nuestro estudio (42 de 83,7\%). Igualmente, existe acuerdo con los diferentes reportes (Baptista et al., 1989; Ortale et al. y Kalpana) sobre el número bajo de casos con dominancia derecha prominente, en las que las ramas ventriculares posteriores de la ACD irrigan los cuadrantes laterales del ventrículo izquierdo.

Tradicionalmente se ha señalado al ápex cardiaco como el sitio donde con mayor frecuencia finaliza la AIA, pero los diferentes reportes, incluido el nuestro, describen la terminación en este segmento solamente entre el 6 al $33 \%$ de los casos. (Sahni \& Jit; Ilia et al.; James, 1961 y Kalpana). Igualmente existe acuerdo entre los anteriores autores que señalan como el sitio más frecuente de finalización al tercio inferior del surco interventricular posterior( $42-80 \%$ ), una vez ha suplido al ápex con numerosas colaterales. La finalización de la RIA antes del ápex es poco frecuente (Sahni \& Jit (2\%); Ilia et al. 1\%; Kalpana $(8 \%)$ y en nuestro trabajo $(1.3 \%))$. James reportó un porcentaje ligeramente mayor (17\%).

La distribución de la RIP en el surco homónimo observada en nuestra serie, es similar a la descrita por James (70 y $30 \%$ para los tipos largo y corto, respectivamente). Debe destacarse que escasamente un tercio de los casos alcanzaron el ápex cardiaco. Kampala encontró en un número significativo de especímenes $(62,5 \%)$ que sólo alcanzaron el nivel de los tercios superior o medio del surco en referencia.

Ante la presencia de RIP cortas se observan fenómenos de compensación en la irrigación de los segmentos posteroinferiores del corazón, dados por el segmento distal de la RIA que, tras superar al ápex, se distribuye en el territorio vecino de la cara diafragmática, irrigando el segmento que no alcanza la RIVP. Esta expresión anatómica debe tenerse en cuenta al evaluar procesos de infarto de miocardio localizados en el segmento inferior de la pared posterior del corazón, en razón a que la obstrucción arterial podría comprometer la porción distal de la rama interventricular anterior, en lugar de la rama interventricular posterior, como suele creerse.

Dodge et al., 1992, Pepine 1998 y Kaimkhani et al. señalan que el calibre de la ACD y de la RCX se afectan por los diferentes tipos de irrigación coronaria. Ellos señalan que el diámetro de la ACD es significativamente menor en el patrón de dominancia izquierda, mientras que el calibre de la RCX es significativamente menor en presencia de dominancia derecha. Pepine y Kaimkhani et al., también encontraron que el diámetro de la arteria coronaria izquierda y de la RIA pueden variar dependiendo del patrón de dominancia. Nosotros reportamos una relación significativa entre el tamaño de ACD y RCX y los patrones de distribución de las arterias coronarias. Nuestros hallazgos están en concordancia con los de Dodge et al., Pepine y Kaimkhani. La causa de estas relaciones probablemente esté dada por complejos procesos embriológicos que determinan simultáneamente el tipo de irrigación y el calibre de los vasos coronarios, los cuales conducen a garantizar una adecuada irrigación de todos los segmentos del corazón. 
La expresión morfológica de la muestra evaluada caracterizada por una dominancia derecha elevada (83.7\%), con predominio del tipo moderado, sumado al número significativo de especímenes con RIVP que finalizan en el tercio medio e inferior del surco homónimo y con meca- nismos angiogénicos de compensación en la cara posterior cardiaca con una RIA que traspasa el ápex, pueden ser características de la irrigación del corazón en la población mestiza colombiana, lo cual debería ratificarse en posteriores estudios.

BALLESTEROS, L. E.; CORZO, E. G. \& SALDARRIAGA, B. Coronary's dominance determination in racially mixed Colombian population. A direct anatomical sudy Int. J. Morphol., 25(3):483-491, 2007.

SUMMARY: There is great variability between results of coronary dominance among several ethnic groups. The purpose of this work was to determine the coronary dominance in a group of half caste Colombian sample. One hundred fifty four hearts obtained from fresh autopsy cadavers were evaluated. Coronary arteries were injected with synthetic resin and later cleaned with $15 \%$ of $\mathrm{KOH}$ for removing the epicardial fat. Right dominance in $83.7 \%$ of the sample with a moderated dominance corresponding to $42.8 \%$ was observed. A balanced circulation in $9.2 \%$ and left dominance in $7.2 \%$ with a major frequency $(3.3 \%$ ) in the subgroup II was observed. Although not statistical significantly different $(\mathrm{p}=0.26)$ we found major female right dominance. Circumflex artery had short and long trajectory in $92.8 \%$ and $7.2 \%$ respectively. Right coronary artery concludes its trajectory between the Cruz cordis and the left margin in $81.8 \%$ and in the left margin in $1.9 \%$. In right coronary dominance the caliper of right coronary arteries was $3.21 \mathrm{~mm}$ (SD 0.53 ) and $2.67 \mathrm{~mm}$ (SD 0.30 ) for the left coronary dominance with an statistical significative difference $(\mathrm{p}=0.0066)$. In right coronary dominance sample there was prevalence of the moderated expression, short circumflex arteries (all dominances) and a major caliper in the right coronary dominance.

KEY WORDS: Heart; Coronary circulation; Dominance, Balanced circulation; Arterial caliper.

\section{REFERENCIAS BIBLIOGRÁFICAS}

Ahmed, S. H.; Al-Rakhawy, M.T. \& Harrison, R.G. A new conception of coronary artery preponderance. Acta Anat., 83:87-94, 1972.

Banchi, A. Morfologia delle arteriae coronariae cordis. Arch. Ital. Anat. Embriol., 3:87-95, 1904.

Baptista, C. A.; DiDio, L. J. \& Teofilovski-Parapid, G. Variation in length and termination of the right coronary artery in man. Jpn. Heart J., 30(6):789-98, 1989.

Baptista, C. A.; DiDio, L. J. \& Teofilovski-Parapid, G. Variation in length and termination of the ramus circumflexus of the human left coronary artery. Anat. Anz., 171(4):247-53, 1990.

Baroldi, G. \& Scomazzoni, G. Coronary circulation in the normal and the pathologic heart. Office of the Surgeon General. Dept of the Army. Washington, D C. 1967.

Blunk, J. N.\& DiDio, L. J. A.Types of coronary circulation in the human hearts. Ohio St. Med. J., 67:596-607, 1971.

Cavalcanti, J. S.; de Lucena Oliveira, M. Pais e Melo, A.V. Jr. \& Balaban, G. Anatomic; variations of the coronary arteries. Arq. Bras. Cardiol., 65(6):489-92, 1995.

De Paula, W. Estudo estadistico sobre a irrigação coronariana no coração humano em brancos e negros. Folha Cli. Biol., 1:20-42, 1972.
DiDio, L. J. \& Wakefield, T.W. Coronary arterial predominance or balance on the surface of the human cardiac ventricles. Anat. Anz., 137(1-2):147-58,1975.

Dodge, J. T. Jr.; Brown, B. G.; Bolson, E. L.\& Dodge, H. T. Lumen diameter of normal human coronary arteries. Influence of age, sex, anatomic variation, and left ventricular hypertrophy or dilation. Circulation, 86(1):23246, 1992.

Hadziselimovic, H. Age characteristics of blood vessels of the human heart. Acta. Anat. (Basel)., 109(3):231-7, 1981.

Falci Junior, R.; Guimaraes, M. H.; Santos, A. P.; Cabral, R. H.; Jatene, F. B.\& de Prates, N. E. Comparative study of the coronary circulation pattern of anatomic specimens and surgical patients. Rev. Hosp. Clin. Fac. Med. São Paulo, 51(6): 224-7, 1996.

James, T. N. Anatomy of the Coronary Arteries. Hoeber, New York, 1961.

James,T. N. Anatomy of the coronary arteries in health and disease. Circulation., 32:1020-33, 1965.

Kaimkhani, Z. A.; Ali, M. M. \& Faruqi, A. M. Pattern of coronary arterial distribution and its relation to coronary artery diameter. J. Ayub. Med. Coll. Abbottabad., 17(1):403, 2005. 
Kalpana, R. A study on principal branches of coronary arteries in humans. J. Anat. Soc. India, 52(2):137-40, 2003.

Loukas, M.; Curry, B.; Bowers, M.; Louis, R. G. Jr.; Bartczak, A.; Kiedrowski, M.; Kamionek, M.; Fudalej, M.\& Wagner, T. The relationship of myocardial bridges to coronary artery dominance in the adult human. Heart. J. Anat., 209(1):43-50, 2006.

Ilia, R.; Rosenshtein, G.; Weinstein, J.; Cafri, C.; Abu-Ful, A.; Gueron, M. Left anterior descending artery length in left and right coronary artery dominance. Coron. Artery. Dis., 12(1):77-8, 2001;

Llerena, L. R.; Llerena, L. D.; Carpio, J. L.\& Gonzalez, A. N. Revisión de 2150 coronariografías. Rev. Cubana. Cardiol. Cir. Cardiovasc., 10(1):67-72, 1996.

May, A. M. Surgical Anatomy of the coronary arteries. Dis. Chest., 38:645-57, 1960.

Mouchet, A. Les artères coronaires du coeur chez l'Homme. $2^{\mathrm{a}}$ ed. Maloine, Paris, 1933.

Nerantzis, C. E.; Antonakis, E. \& Avgoustakis,A. A new corrosion casting technique. Anat. Rec., 191:321- 6,1978.

Nerantzis, C. E.; Papachristos, J. C.; Gribizi, J. E.; Voudris, V. A.; Infantis, G. P.; Koroxenidis, G.T. Functional dominance of the right coronary artery: incidence in the human heart. Clin. Anat., 9(1):10-3, 1996.

Ortale, J. R.; Keiralla, L. C. \& Sacilotto, L. The posterior ventricular branches of the coronary arteries in the human heart. Arq. Bras. Cardiol., 82(5):468-72, 2004.

Penthe, P.; Bara, J. A. \& Blanc, J. J .Etude anatomique descriptive des gros troncs coronariens et des principales collaterales epicardiques. Nouv. Presse. Med., 5:715,1976 .

Pepine, C. J. Coronary angiography catheterization. In: Topol E. J. editor. Topol Texbook of cardiovascular Medicine. Philadelphia, WB Saunders Company, 1998. pp.710-11.

Pessa, C. J.; Gomes, W. J.; Catani, R. \& Prates, J. C. Anatomical relationships between the posterior mitral valve annulus and the coronary arteries. Implications to operative treatment. Braz. J. Cardiovasc. Surg., 19(4): 372-7, 2004.

Polacek, P. \& Zechmeister, A. The occurrence and significance of myocardical bridges and loops on coronary arteries. University. J. E. Purkyne., 10(2):125-31, 1958.
Reig, J.; Longan, S. \& Domenech, J. M. The circumflex branch of the left coronary artery in the human infant. $J$. Anat., 155:1-10, 1987.

Reig, J.; Jornet, A. \& Petit, M. Patterns of the coronary artery irrigation in the left ventricle. Surg. Radiol. Anat., 15(4):309-14, 1993.

Sahni, D. \& Jit, I. Blood supply of the human interventricular septum in north-west Indians. Indian. Heart. J., 42(3):161-9, 1990.

Schlesinger, M. J. Relation of anatomic pattern to pathologic conditions of the coronary arteries. Arch. Pathol., 30: 403-15, 1940.

Taylor, J. R. Short left circumflex artery. Arch. Pathol. Lab. Med., 101(2):83-5, 1977.

Zbigniew, K. \& Mikusek, J. Clinical and morphological studies on varieties of coronary vascularisation of diaphragmatic surface of human heart. Med. Sci. Monit., 6(2):253-7,2000.

\section{Dirección para correspondencia: \\ Dr. Luis Ernesto Ballesteros \\ Calle 108 21A-13. \\ Bucaramanga \\ COLOMBIA}

Teléfono: 57-097-6344000 ext. 3124

Fax: 57-097-6455693

Email: Iballest56@yahoo.es

Recibido : 10-05-2007

Aceptado: 12-06-2007 
